\title{
The Infrared Companions of T Tauri Stars: Clues to the Formation and Early Evolution of Binaries
}

\author{
Chris D. Koresko \\ Jet Propulsion Laboratory, M/S 171-113, Pasadena, CA 91109 \\ Christoph Leinert \\ Max-Planck-Institut für Astronomie, Königstuhl 17, D-69117 \\ Heidelberg, Germany
}

\begin{abstract}
Infrared companions are young stellar objects with unusual properties gravitationally bound to more or less typical T Tauri stars. As such they promise to be the source of information on either a particular phase in the development of young stars or on a particular mode of development. We discuss the observed properties of infrared companions as well as attempts to explain their physical status with the aim to see how much of solid conclusion has been obtained so far.
\end{abstract}

\section{Introduction}

In the most favored picture of binary and multiple star formation, the various stellar components are produced when a collapsing cloud core fragments under the influence of rotation, magnetic fields, and turbulence (c.f., a number of papers in this volume). A basic prediction of this picture is that the stellar components are coeval. From that point onward, they evolve nearly independently at predictable rates determined by the physics of convection, nuclear burning, and radiative transfer in their interiors. A comparison of the evolutionary ages of the components of a given system at any time after its formation should yield consistent results.

The pioneering work of Hartigan et al. 1994 showed that this prediction holds true for $2 / 3$ of the 26 wide (projected separation $400-6000 \mathrm{AU}$ ) premain sequence visual binaries which composed their sample. When there were significant age differences between the component stars, the less massive star was usually the younger. More recent observations by Brandner \& Zinnecker (1997) showed that all 8 of their sample of binaries with projected separations between 85 and $240 \mathrm{AU}$ were consistent with coeval formation. Woitas \& Leinert (2000) reach similar conclusions about a set of 17 weak-lined TTS (WTTS) binaries, but they also find some unusually red objects in a sample of the more extreme classical T Tauri stars (CTTS).

The red objects described by Woitas \& Leinert (2000) are candidate members of a class of $\mathrm{T}$ Tauri companions which may present a significant challenge to the fragmentation picture. These objects are referred to as the "Infrared Companions" (IRCs). They radiate predominantly in the infrared, giving them 
a very youthful appearance which seems inconsistent with the ages of the stars they orbit. Zinnecker \& Wilking (1992) estimate that IRCs constitute perhaps $10 \%$ of all $\mathrm{T}$ Tauri binary companions detected in the binary surveys of Ghez, Neugebauer, \& Matthews (1993) and Leinert et al. (1993). The samples in these studies contained primarily CTTS in the Taurus and Ophiuchus star-forming regions (SFRs). A list of several of the best examples of IRCs is given in Table 1.

\begin{tabular}{llll}
\multicolumn{1}{c}{ Table 1. } & \multicolumn{2}{c}{ Selected Infrared Companions } & \\
\hline System & Discovered & by & Technique \\
\hline UY Aurigae & 1944 & Joy \& van Biesbroek & Visible Image \\
T Tauri & 1982 & Dyck, Simon, \& Zuckerman & IR speckle \\
VV CrA & 1985 & Frogel & IR offset \\
DoAr 24e & 1988 & Chelli et al. & IR speckle \\
Glass I & 1988 & Chelli et al. & IR speckle \\
Haro 6-10 & 1989 & Leinert \& Haas & IR speckle \\
XZ Tauri & 1990 & Haas, Leinert, \& Zinnecker & IR speckle \\
\hline \hline
\end{tabular}

The most basic question that will be addressed here is this: Are the IRCs truly stars with significantly younger evolutionary ages than their primaries, or are they more evolved (presumably coeval) objects whose appearance has been modified by some process which alters either the light they radiate or the stars themselves? If the latter, what are the implications of the existence of such a process for our understanding of the evolution of multiple stars, and for stars in general?

\section{Properties of the Infrared Companions}

While the IRC class is defined by bright infrared and faint visible radiation and the presence of a $\mathrm{T}$ Tauri primary, a multitude of observations over the years since the discovery of the first IRC (the T Tauri IRC, by Dyck, Simon, \& Zuckerman 1982) have identified a number of other unusual and perhaps characteristic features of these objects. For the most part, these features tend to be more extreme versions of phenomena seen in more "normal" T Tauri stars, such as infrared excesses, photometric variability, and shock-excited emission lines of molecular hydrogen. Other features such as rapidly-changing nonthermal radio emission seem to be more or less unique to the IRC class among the CTTS or even to specific IRCs. Here we discuss these features in some detail.

\subsection{Infrared Excess and Bolometric Temperature}

It is useful to examine scalar quantities which can be calculated from the SED to estimate the importance of the infrared excess in an IRC. Here we examine two such quantities which have been applied in the literature to various young stellar objects. The simplest is perhaps the fraction $L_{\mathrm{IRAS}} / L_{\mathrm{bol}}$ of the object's total luminosity which emerges in the IRAS passbands at wavelengths between 12 and $100 \mu \mathrm{m}$. For even a very cool stellar photosphere, this ratio is less than 0.01 , while for stars which are "embedded" according to the definition of Kenyon, Calvet, \& Hartmann (1993) it is at least 0.8. This latter value corresponds to a blackbody temperature of $220 \mathrm{~K}$. The IRCs studied by Koresko, Herbst, \& Leinert (1997; hereafter KHL) do not quite reach this degree of "embeddedness", 


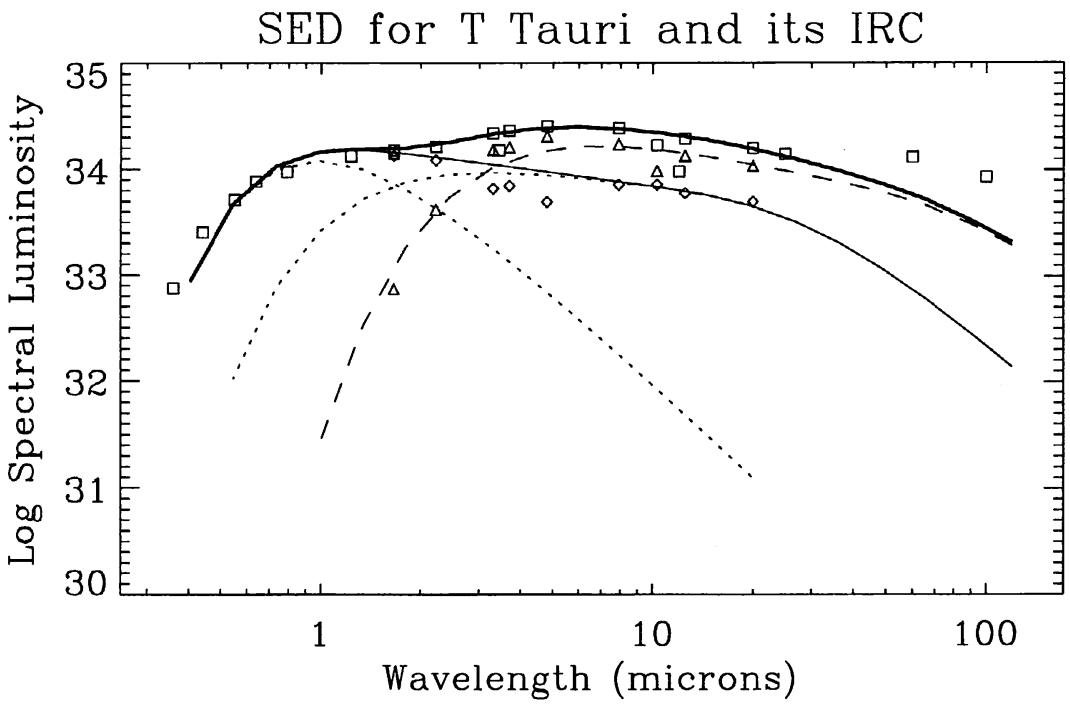

Figure 1. The SED for the components of the T Tauri system are plotted along with with simple models. The squares trace the total flux of the system, while the diamonds and triangles mark the fluxes of the primary and IRC, respectively. The data and models are from KHL and references therein.

having $L_{\mathrm{IRAS}} / L_{\mathrm{bol}}$ between 0.18 (for DoAr 24E IRC) and 0.76 (for Haro 6-10 IRC).

On a plot of the bolometric luminosity $\mathrm{L}_{\text {bol }}$ versus bolometric temperature $\mathrm{T}_{\text {bol }}$ (Myers and Ladd, 1993), the IRCs are clustered approximately midway between the warmest embedded sources and the coolest CTTS. The bolometric temperatures of the sample of IRCs studied by KHL range from 210 to $800 \mathrm{~K}$.

\subsection{Primary Stars}

The IRCs are by definition associated with more or less "normal" T Tauri stars, which in practice are usually $\sim 10^{6}$ yr old CTTS with significant infrared excesses of their own. For the present, we will refer to these visibly-bright stars as the "primaries" regardless of their relative bolometric luminosities, following the convention of KHL.

\subsection{Spectral Properties}

The availability of visible-light spectra of IRCs is limited at present because of their faintness and proximity to primaries which are much brighter at visible wavelengths. The Glass I IRC is an exception, perhaps because it was in visuallybright phase at the time of the spectral observations. Feigelson \& Kriss (1989) obtained visual spectra of the primary and the IRC, for which they estimated spectral types of K3 and G5e, respectively. And Reipurth \& Zinnecker (1993) 
in their imaging survey for young binaries, found the Glass I IRC to be nearly as bright as the primary in the Gunn $\mathrm{z}$ filter.

Unlike the visual spectra, infrared spectral information (albeit at low resolution) is relatively plentiful. Herbst, Koresko, \& Leinert (1995; hereafter HKL) obtained $\mathrm{R} \sim 250$ spectra in the $\mathrm{K}(2.0-2.4 \mu \mathrm{m})$ band of the primaries and companions in the UY Aurigae and Haro 6-10 system. They found that the primary stars displayed normal photospheric absorption features roughly consistent with the temperatures determined from visible-light spectra, together with the $\mathrm{Br} \gamma$ emission line of atomic hydrogen which is common in T Tauri stars. The spectrum of the UY Aurigae IRC was similar to that of its primary star except for the presence of a $\mathrm{v}=1-0 \mathrm{~S}(1)$ emission line of molecular hydrogen, while the Haro 6-10 IRC displayed only a red continuum which was featureless except for the same molecular hydrogen line.

Mid-infrared spectra of the IRCs orbiting T Tauri and Haro 6-10 (Ghez et al. 1991; van Cleve et al. 1994; Herbst, Robberto, \& Beckwith 1997) showed deep $10 \mu \mathrm{m}$ silicate absorption features which are not seen in their primaries, indicating that they suffer strong extinction due to localized dust distributions. The $\mathrm{T}$ Tauri primary appears to show the same silicate feature in emission. Herbst, Robberto, \& Beckwith (1997) found that the shapes of the silicate features in both the primary and the IRC could be reproduced with a model in which each star is surrounded by a disk which has tenuous upper layers of varying emissivity and density.

It cannot be said that the water ice absorption feature at $3.09 \mu \mathrm{m}$, which is well correlated with visual extinction $\mathrm{A}_{V}$ (Whittet et al. 1988), is unusually common in infrared companions. But in Haro 6-10 (alias Elias 7) Whittet et al. (1988) found strong ice band absorption. It was an independent confirmation for the large amount of circumstellar dust around the infrared companion in this binary that spatially resolved observations (Leinert et al. 1996; Beck et al. 2000; Leinert, Ligori, \& Woitas 2000, hereafter LLW) showed the ice feature to be concentrated on the companion.

\subsection{Photometric and Spectral Variability}

Strong photometric variability appears to be common, and perhaps universal, among IRCs. At least five IRC systems are known to exhibit photometric variations. The most extreme example known at present is UY Aurigae, whose IRC was originally discovered as a visible star only 0.5 mag fainter than its primary (Joy \& van Biesbroeck 1944). Half a century later, a much more sensitive Rband CCD image failed to detect the object at all, indicating that the IRC had become at least 5 magnitudes fainter than the primary (HKL).

The IRC system with the best-sampled light curve is probably Haro 6-10, with photometric monitoring available (LLW; Beck et al. 2000) with sufficient angular resolution to derive the brightness of the IRC at near-infrared wavelengths over a total timespan of more than a decade, with unresolved near-infrared photometry of the system being available for another 14 years. Both primary and IRC exhibit significant and irregular variability in both brightness and color across the near-infrared region, with the IRC's variability being generally larger than the primary's. The colors of the stars usually seem to be unrelated to the brightness of the primary, and the variations of the two stars are usually uncor- 
related with each other. This leads LLW to conclude that a single mechanism such as changes in reddening is inadequate to explain the observed behavior of the system. Variations in the ice feature provide perhaps the most direct clue to the varying distribution of dust (Beck et al. 2000; LLW), but no clear picture has yet evolved from these observations.

The T Tauri IRC went through an outburst between 1989-91 in which it brightened by $\sim 2$ magnitudes between 2 and $10 \mu \mathrm{m}$ (Ghez et al. 1991; Kobayashi, et al. 1994; Simon et al. 1996). Roddier et al. (2000) found that the magnitude difference between the primary and IRC in the $\mathrm{K}$ band changed from 2.6 on Christmas 1994 to 0.6 in November 23, 1999, and monitoring by Beck et al. (2000) over a two-year period ending in January 2000 showed changes as large as $50 \%$ in its $\mathrm{K}$-band flux measured on dates only a few days apart. By contrast with the IRC and also with the Haro 6-10 primary, the T Tauri primary appears to be relatively stable during the Beck et al. (2000) period.

The brightness ratios of the IRC systems UY Aurigae, Do-Ar 24E, and VV CrA have also been observed to vary substantially in the near-infrared (KHL and references therein).

\subsection{Infrared Morphology}

In recent years the angular resolution available for near-infrared imaging observations has been improved significantly due to the development of speckle and adaptive optics capabilities for telescopes with apertures as large as $10 \mathrm{~m}$. These facilities have made it possible to begin to resolve structure in some of the IRCs, and thereby directly test some of the models.

The most-studied object so far is the T Tauri IRC. A series of careful observations using adaptive optics first confidently detected resolved structure in this object (Roddier et al. 2000) which was later found to be a pair of pointlike stars separated by $0^{\prime \prime} .05$, corresponding to a projected separation of $7 \mathrm{AU}$ at the distance to the Taurus SFR (Koresko 2000). Followup adaptive-optics compensated speckle interferometry (AOCSI) observations have detected a change in the separation and position angle of the IRC double which is consistent with orbital motion (Köhler, Kasper, \& Herbst 2000). The duplicity of the T Tauri IRC may explain why, despite the large extinction, submillimeter images place upper limits on its circumstellar dust mass no more than $3 \times 10^{-3} \mathrm{M}_{\odot}$, which is well below the mass of the disk around the primary star (Hogerheijde et al. 1997; Akeson et al. 1998).

In contrast with T Tauri, holographic observations of the Haro 6-10 IRC revealed a bright, compact, nebulous object which shows no sign of duplicity (Koresko et al. 1999). Holography of the IRC in the VV CrA system has detected an extended envelope which contributes $\sim 10 \%$ of the total light at $2.2 \mu \mathrm{m}$, and no evidence for a tertiary companion (Koresko et al. 2001). Finally, holography observations of the IRCs of Do-Ar 24E, UY Aurigae, and WSB 4 show no sign of extended structure at all (Koresko et al. 2001).

\subsection{Radio Emission and Circumstellar Matter}

In a seminal study, Beckwith et al. (1990) surveyed a sample of $86 \mathrm{~T}$ Tauri stars in the radio continuum at $1.3 \mathrm{~mm}$, with the goal of detecting and measuring the thermal emission from dust in circumstellar disks. The submillimeter survey 
sample included the IRC binaries T Tauri, UY Aurigae, and XZ Tau. These systems do not stand out among the sample as having unusually large dust masses. In particular, the dust masses are always small compared to the stellar masses, confirming that the IRCs have evolved beyond the phase in which their masses grow significantly via accretion.

Although a number of pre-main sequence stars are radio emitters (e.g., Chiang, Phillips \& Lonsdale 1995 and references therein), the T Tauri IRC is one of only two known pre-main sequence sources of circularly polarized nonthermal radiation at centimeter wavelengths (Phillips et al. 1993; Skinner \& Brown 1994), the other being the Class 1 protostar IRS 5 in the Corona Australis "Coronet Cluster" (Feigelson, Carkner, \& Wilking 1998). This observation hints at the action of some unusual energetic process, perhaps accretion-driven, involving strong magnetic fields.

\section{The Nature of the IRC Phenomenon}

A number of questions arise immediately when one considers the observational data on IRCs. Are these objects fundamentally different from their primary stars, with their infrared excesses and activity pointing to an evolutionary phase younger than the T Tauri stars, or do their youthful outward appearances hide more mundane central stars? Is there an "IRC phase" which most or all T Tauri stars in binaries go through, or does the IRC phenomenon occur only for some specific range of stellar parameters and environment? Is there a favored binary separation range? What about single T Tauri stars? If the IRCs are episodic accreters, how long do the episodes last, how many will occur during the premain sequence lifetime of the star, and how much mass is gained during these episodes?

We are not yet in a position to answer many of these questions in detail. But it does make sense to begin attempting to answer a few of the basic questions about the nature of the IRCs, and to explore the extent to which current observational can constrain the models.

\subsection{Are the IRCs Coeval with their Primaries?}

The combination of large and possibly anisotropic extinctions, infrared excesses, variability, and faintness at visible wavelengths, have made it difficult or impossible to derive reliable age estimates for the IRCs. Despite these difficulties, a few attempts have been made to test IRCs for coevality with the primaries, with results that seem to support coeval formation. KHL assumed coevality and placed each of the six IRCs in their sample on an H-R diagram to derive estimates for their effective temperatures. The result for the Glass I IRC was consistent with the G5e spectral type estimated by Feigelson \& Kriss (1989) from a visible-light spectrum taken in 1981, when the IRC was a visible CTTS with $\mathrm{m}_{\mathrm{V}}=14.09$. For the UY Aurigae IRC, KHL found effective temperatures corresponding to a spectral type of $\mathrm{K} 6-\mathrm{K} 7$, which is roughly consistent with the M0 roughly estimated from the low-resolution near-infrared spectrum of HKL. Close et al. (1998) fit models of extincted stars surrounded by warm disks to the SEDs of UY Aurigae and its IRC, and found that they could be fit with a common age of $3 \times 10^{5} \mathrm{yr}$ and HKL's spectral types of K7 and M0. 


\section{2. $\quad$ Special Viewing Geometries}

Perhaps the simplest model for an IRC would be a normal T Tauri star positioned behind normal disk material around the primary or the IRC itself. In this picture, the IRCs could be intrinsically quite similar to their primaries. Here we briefly discuss two models that have been proposed in the literature.

For example, the IRCs could be surrounded by circumstellar disks viewed nearly edge-on. The extinction would be produced by the material in the outer disk, which is perhaps inhomogeneous so that the dust column varies significantly as orbital motion carries different parcels of mass through the line of sight. However, none of the IRCs show a morphology similar to that of the $100 \mathrm{AU}$ disk structure surrounding the companion to HK Tauri (Koresko 1998). Further, the high extinction to the hot inner regions of the HK Tauri disk make the shape of the system's SED is more reminiscent of a moderately-extincted star than of an IRC. However, it is still possible that certain combinations of small disk mass and orientations not quite edge-on can reproduce the observations. Detailed radiative-transfer modeling is needed to fully test these possibilities.

A second possibility is that the IRCs lie behind the disks associated with their primary stars, as has been suggested for the T Tauri system (e.g., van Langevelde et al. 1994). As noted by Akeson et al. (1998), although the radius of the $\mathrm{T}$ Tauri $\mathrm{N}$ disk appears smaller in their submillimeter images than the distance to the IRC, submillimeter imaging cannot rule out the existence of a more diffuse outer disk such as that proposed by Hogerheijde et al. (1997).

\subsection{Disk Accretion}

It has been proposed that the origin of the large and variable infrared excess in the T Tauri IRC is an active accretion disk (Ghez et al. 1991). This is sometimes referred to as the "mini-FUor" model, after its similarity to the FU Orionis class of objects which are believed to be T Tauri stars whose disks are undergoing episodes of very rapid accretion. The IRCs would be considerably less extreme cases, in which the luminosity due to the disk is never very much larger than that of the stellar photosphere.

The mini-FUor model was elaborated on by KHL, who suggested that the accretion could be triggered by gravitational interactions, and synchronized with the orbital phase, if the binary orbits are eccentric. A particularly interesting possibility is that the IRC phenomenon is both triggered and fueled by streams of matter coming from a circumbinary disk, as predicted by hydrodynamical models (Artymowicz \& Lubow 1996). Alternatively, surges of accretion and extinction may be explained if the IRC systems are disintigrating triple systems with associated dusty envelopes (Reipurth 2000). The unstable orbits of such a system may cause the lighter components to be found at large radii and suffer small extinction while the more massive and luminous objects lie deeper within the envelope, suffering larger extinction, while their accretion could be accelerated by infall and/or perturbations of the disk.

The T Tauri and Haro 6-10 IRCs are suspected to drive the giant HerbigHaro flows which have been seen to extend from somewhere in these binaries to distances of $\sim 1$ pc (Solf \& Böhm 1999; Reipurth, Bally, \& Devine 1997; Movsessian \& Makagian 1999; Devine et al. 1999). The presence of such flows may indicate rapid accretion in these objects. 


\subsection{Episodic Accretion}

In the KHL picture, one can make some very crude and unreliable estimates of the frequency and duration of a typical IRC event in the recurrent "episodic accretion" picture in the following way: Starting with the estimate that $10 \%$ of the companions of classical T Tauri stars are IRCs (Zinnecker \& Wilking 1992) and the assumption that these episodes occur periodically with constant frequency and duration for all CTTS companions throughout a typical pre-main sequence lifetime of $10^{6} \mathrm{yr}$, one finds that each object spends a total of $10^{5}$ $\mathrm{yr}$ as an IRC. If exactly one among the half dozen or so well-studied IRCs has changed state from non-IRC to IRC in the last $\sim 25 \mathrm{yr}$, then for each object these transitions occur on timescales of $\sim 150 \mathrm{yr}$. Considering the crudeness of this calculation, this timescale is more or less consistent with the orbital timescale of $\sim 700 \mathrm{yr}$ for a pair of solar-mass stars separated by $100 \mathrm{AU}$.

The infall of $10^{-7} \mathrm{M}_{\odot} \mathrm{yr}^{-1}$ of gas onto a solar-mass star of radius $3 \mathrm{R}_{\odot}$ would be required to produce the $1 \mathrm{~L}_{\odot}$ typical of an IRC's infrared excess. At this rate, and using the above estimate for the total time spent as an IRC, a typical T Tauri companion would accrete $10^{-2} \mathrm{M}_{\odot}$. This mass is comparable to the mass of a typical pre-main sequence disk.

Clearly, these results do not demonstrate the validity of the recurrent episodic-accretion picture for the IRCs, but they do show that it is not grossly inconsistent with the observations.

\subsection{Binary Separations}

The angular separations between the known IRCs and their primaries are typically between $0^{\prime \prime} .3$ and $3^{\prime \prime} .0$, corresponding to projected linear distances of $\sim 40-400 \mathrm{AU}$. These distances are close to the fiducial size of a circumstellar disk, suggesting that star/disk interactions might play a role in triggering and/or fueling the IRC phenomenon. Unfortunately, the significance of this distance may be obscured by observational effects: Historically, it has been difficult to measure the photometric fluxes of individual stars with separations smaller than this range, and at separations a few times larger it becomes uncertain whether the two stars form a true binary pair. The separation range of the known IRC binaries may therefore be strongly biased by selection effects.

\section{Implications for the Formation and Evolution of Binary Stars}

At the present time, the existence of the IRCs, whose ages are possibly inconsistent with coeval formation, represent a potential challenge to the fragmentation scenario for the formation of binary stars. However, this challenge appears to be muted by the fact that the two IRCs whose ages have been studied seem consistent with their primaries. It now seems likely that the IRC phenomenon neither demands nor produces large deviations from normal pre-main sequence evolution. This tentative conclusion will need to be reexamined as improved observations become available.

On the other hand, it is very likely that the interactions between stars and disks in binary systems will have profound effects, if not on the stars, then on the disks and whatever planetary systems may come into being as they dissipate. 
The IRCs, with their large infrared excesses and other signs of unusually strong activity, may ultimately be tracers of these interactions, and their study an important means to understanding how planets may evolve in the most common stellar environments: binary stars.

\section{References}

Akeson, R. L., Koerner, D. W., \& Jensen, E. L. N. 1998, ApJ, 505, 358

Artymowicz, P., \& Lubow, S. H. 1996, ApJ, 467, L77

Beck, T. L., Simon, M., Ghez, A. M., Prato, L., Howell, R. R. this volume

Beckwith, S. V. W., Sargent, A. I., Chini, R. S., \& Guesten, R. 1990, AJ, 99, 924

Brandner, W., \& Zinnecker, H. 1997, A\&A, 321, 220

Chen, H., Myers, P. C., Ladd, E. F., \& Wood, D. O. S. 1995, ApJ, 445, 377

Chiang, E., Phillips, R. B., \& Lonsdale, C. J. 1996, AJ, 111, 355

Close, L. M., Dutrey, A., Roddier, F., Guilloteau, S., Roddier, C., Northcott, M., Menard, F., Duvert, G., Graves, J. E., Potter, D. 1998, ApJ, 499, 883

Devine, D., Reipurth, B., Bally, J., \& Balonek, T. J. 1999, AJ, 117, 2931

Dyck, H. M., Simon, T., \& Zuckerman, B. 1982, ApJ, 255, L103

Feigelson, E. D., \& Kriss, G. A. 1989, ApJ, 338, 262

Feigelson, E. D., Carkner, L., \& Wilking, B. A. 1998, ApJ, 494, L215

Ghez, A. M., Neugebauer, G., Gorham, P. W., Haniff, C. A., Kulkarni, S. R., Matthews, K., Koresko, C., \& Beckwith, S. 1991, AJ, 102, 2066

Ghez, A. M., Neugebauer, G., \& Matthews, K. 1993, AJ, 106, 2005

Hartigan, P., Strom, K. M., \& Strom, S. E. 1994, ApJ, 427, 961

Herbst, T. M., Koresko, C. D., \& Leinert, C. 1995, ApJ, 444, L93 (HKL)

Herbst, T. M., Robberto, M., Beckwith, S. V. W. 1997, AJ, 114, 744

Hogerheijde, M. R., van Langevelde, H. J., Mundy, L. G., Blake, G. A., \& van Dishoeck, E. F. 1997, ApJ, 490, L99

Joy, A. H., \& an Biesbroeck, G. 1944, PASP, 56, 123

Kenyon, S. J., Calvet, N., \& Hartmann, L. 1993, ApJ, 414, 676

Kobayashi, N., Nagata, T., Hodapp, K., \& Hora, J. L. 1994, PASJ, 46, L183

Köhler, R., Kasper, M., Herbst, T., this volume

Koresko, C. D., Herbst, T. M., \& Leinert, C. 1997, ApJ, 480, 741 (KHL)

Koresko, C. D. 1998, ApJ, 507, L145

Koresko, C. D., Blake, G. A., Brown, M. E., Sargent, A. I., \& Koerner, D. W. 1999, ApJ, 525, L49

Koresko, C. D. et al. 2001, in preparation

Leinert, C., Zinnecker, H., Weitzel, N., Christou, J., Ridgway, S. T., Jameson, R., Haas, M., \& Lenzen, R. 1993, A\&A, 278, 129 
Leinert, Ch., Haas, M., Weitzel, N., in Disks and Outflows around Young Stars, ed. S. V. W. Beckwith, J. Staude, A. Quetz \& A. Natta, Lecture Notes in Physics 465, Springer, Berlin 2000, CD-ROM, 411-414

Leinert, Ch., Ligori, S., Woitas, J., this volume (LLW)

Myers, P. C., \& Ladd, E. F. 1993, ApJ, 413, L47

Movsessian, T. A., \& Magakian, T. Y. 1999, A\&A, 347, 266

Phillips, R. B., Lonsdale, C. J., \& Feigelson, E. D. 1993, ApJ, 403, L43

Reipurth, B. 2000, this volume

Reipurth, B., Zinnecker, H. 1993, A\&A, 278, 81

Reipurth, B., Bally, J., \& Devine, D. 1997, AJ, 114, 2708

Roddier, F., Roddier, C., Brandner, W., Charissoux, D., Véran, J. -P., \& Courbin, F. 2000, IAU Symposia, 200, Poster Proc., 60

Skinner, S. L., \& Brown, A. 1994, AJ, 107, 1461

Simon, M., Longmore, A. J., Shure, M. A., \& Smillie, A. 1996, ApJ, 456, L41

Solf, J., \& Böhm, K. -H. 1999, ApJ, 523, 709

van Cleve, J. E., Hayward, T. L., Miles, J. W., Gull, G. E., Schoenwald, J., \& Houck, J. R. 1994, Ap\&SS, 212, 231

van Langevelde, H. J., van Dishoeck, E. F., van der Werf, P. P., \& Blake, G. A. 1994, A\&A, 287, L25

Whittet, D. C. B., Bode, M. F., Longmore, A. J., Adamson, A. J., McFadzean, A. D., Aitken, D. K., Roche, P. F. 1988, MNRAS, 233, 321

Woitas, J., Leinert, Ch., Köhler, R. 2000, submitted to A\&A

Zinnecker, H., \& Wilking, B. A. 1992, Binaries as Tracers of Stellar Formation, ed. A. Duquennoy \& M. Mayor, Cambridge University Press, ISBN \# 0521433584, 269

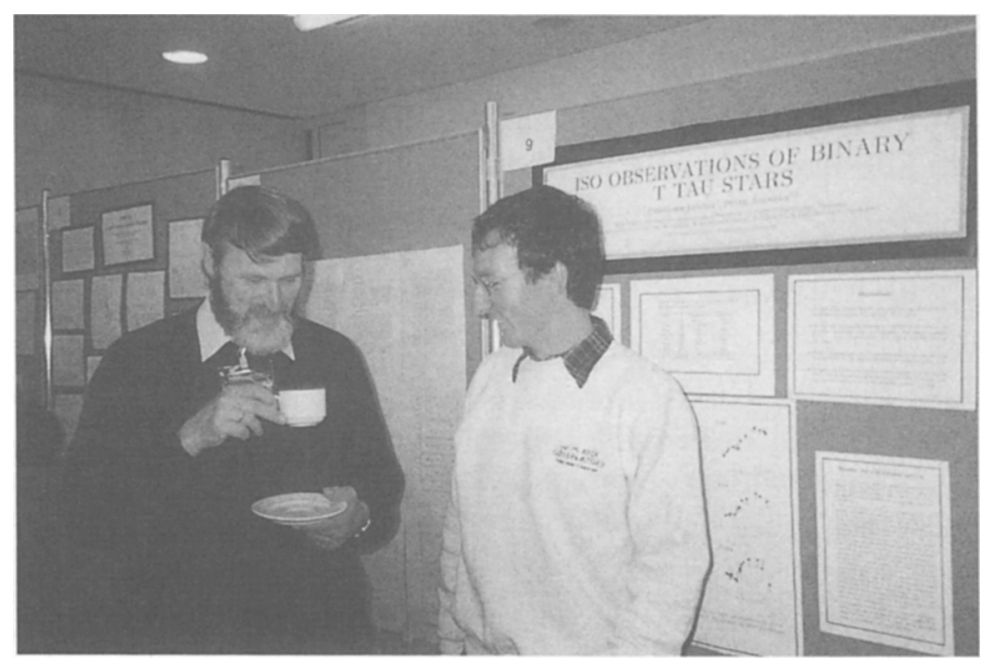

Christoph Leinert, Chris Koresko 